\title{
Noise Equivalent Circuit of a Semiconductor Laser Diode
}

\author{
CHRISTOPH HARDER, JOSEPH KATZ, S. MARGALIT, J. SHACHAM, AND AMNON YARIV, FELLOW, IEEE
}

\begin{abstract}
The noise equivalent circuit of a semiconductor laser diode is derived from the rate equations including Langevin noise sources. This equivalent circuit allows a straightforward calculation of the noise and modulation characteristics of a laser diode combined with electronic components. The intrinsic junction voltage noise spectrum and the light intensity fluctuation of a current driven laser diode are calculated as a function of bias current and frequency.
\end{abstract}

\section{INTRODUCTION}

$\mathbf{M}$ ODELING of semiconductor laser diodes combined with other electronic components requires an accurate representation of the laser. As a first step, a small-signal electrical circuit of a laser has been developed [1] , [2]. The purpose of this work is to extend the model to include noise sources. The extended equivalent circuit allows a straightforward calculation of the modulation and noise characteristics of a laser in dependence of the extrinsic elements, such as the driving source and the parasitic elements.

Intrinsic intensity fluctuations in semiconductor laser diodes are caused by quantum-statistical photon generation and electron-hole recombination within the lasing medium. The intensity noise spectrum for laser light was first calculated by McCumber for a four-level laser system using rate equations with Langevin noise sources [3]. A quantum-mechanical justification of such an approach has been given by Lax [4]. Later, this model was adapted to semiconductor lasers [5], [6]. In early GaAs diode lasers the intrinsic light fluctuations were masked by excess noise due to inadequate transverse mode control, but measurements in stripe contact lasers were nevertheless able to confirm the basic features of the theory, namely large fluctuations at the onset of stimulated emission and a high-frequency noise peak at the resonance frequency of the laser [7].

Recently, it has been verified experimentally [8], [9] that mode stabilized lasers, such as the channeled-substrate planar and the buried heterostructure, operate at the quantum noise limit in excellent agreement with McCumber's noise theory.

Manuscript received August 24, 1981; revised October 29, 1981. This work was supported by the Office of Naval Research, the National Science Foundation under the Optical Communication Program, and the Army Research Office.

C. Harder, S. Margalit, and A Yariv are with the California Institute of Technology, Pasadena, CA 91125.

J. Katz is with the Jet Propulsion Laboratory, California Institute of Technology, Pasadena, CA 91109.

J. Shacham is with Technion-Israel Institute of Technology, Haifa, Israel.
These intrinsic intensity fluctuations are usually very small and the noise performance of an optical communication system is normally determined by the receiver, but in some specific applications the quantum noise may significantly reduce the signal-to-noise ratio $[10]$.

\section{Intensity Noise of A Single-Mode LASER Diode}

The spectral intensity of the quantum fluctuations of the photon and electron population can be found by adding to the familiar rate equations Langevin source terms for electrons $f_{N}(t)$ and for photons $f_{S}(t)$, assumed to have shot noise character [3] , [5].

$$
\begin{aligned}
& \frac{d n}{d t}=\frac{i}{q}-\frac{n}{\tau_{s}}-\left(E_{C V}-E_{V C}\right) s+f_{N} \\
& \frac{d s}{d t}=\left(E_{C V}-E_{V C}\right) s+\beta \frac{n}{\tau_{s}}-\frac{s}{\tau_{p h}}+f_{S} .
\end{aligned}
$$

$n$ and $s$ are the total number of electrons in the gain medium and photons in the single lasing mode. The pump rate is $i / q$, $\tau_{s}$ is the spontaneous and $\tau_{p h}$ the photon lifetime. $E_{C V} s$ is the downward stimulated rate (stimulated emission) and $E_{V C}$ $s$ is the upward stimulated transition rate (stimulated absorption). The gain $g$, which is by definition the net stimulated emission, is $g=E_{C V}-E_{V C} . \quad \beta n / \tau_{s}$ is the spontaneous emission coupled into the lasing mode. By setting $\left\langle f_{N}\right\rangle=\left\langle f_{S}\right\rangle=0$, where \langle\rangle denotes the time average, the equations for the average rates are obtained

$$
\begin{aligned}
\frac{i_{0}}{q}-\frac{n_{0}}{\tau_{s}}-g_{0} s_{0} & =0 \\
-\frac{s_{0}}{\tau_{p h}}+g_{0} s_{0}+\beta \frac{n_{0}}{\tau_{s}} & =0 .
\end{aligned}
$$

For a given dc pump current $i_{0}$ and gain dependence on injected carriers $g(n),(2 a)$ and $(2 b)$ allow us to calculate the resulting average electron population $n_{0}$, average gain $g_{0}=$ $g\left(n_{0}\right)$, and the average number of photons $s_{0}$ in the lasing mode.

Following the treatment of McCumber [3], each change in the photon or electron population is associated with a noise impulse of unit integrated intensity. The spectral intensities of the Langevin noise sources $f_{N}$ and $f_{S}$ and the cross spectral density are therefore given by the shot noise expression [11], [12] and can be written by inspection of the particle rates entering and leaving the electron and photon reservoir as displayed in Fig. 1. 


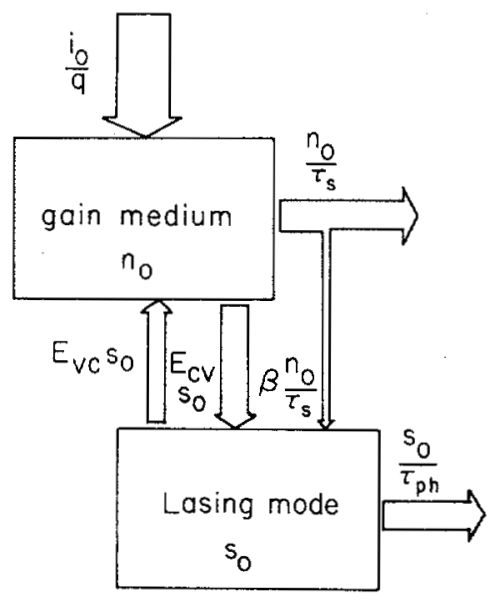

Fig. 1. Model for the shot noise of a semiconductor laser. Each change in the electron $\left(n_{0}\right)$ or photon $\left(s_{0}\right)$ population is associated with a noise impulse of unit integrated intensity.

$$
\begin{aligned}
\left\langle F_{N}^{2}(f)\right\rangle & =\sum r_{n}^{+}+\sum r_{n}^{-}=E_{V C s_{0}}+E_{C V} s_{0}+\frac{n_{0}}{\tau_{s}} \\
& =\frac{i_{0}}{q}+2 E_{V C} s_{0} \\
\left\langle F_{S}^{2}(f)\right\rangle & =\sum r_{s}^{+}+\sum r_{s}^{-} \\
& =\beta \frac{n_{0}}{\tau_{s}}+E_{V C} s_{0}+E_{C V s_{0}}+\frac{s_{0}}{\tau_{p h}} \\
& =2 \frac{s_{0}}{\tau_{p h}}+2 E_{V C} s_{0} \\
\left\langle F_{S}(f) F_{N}(f)\right\rangle & =-\left[\sum r_{n s}+\sum r_{s n}\right] \\
& =-\left[E_{C V} s_{0}+\beta \frac{n_{0}}{\tau_{s}}+E_{V C s_{0}}\right] \\
& =-\left[\frac{s_{0}}{\tau_{p h}}+2 E_{V C} s_{0}\right]
\end{aligned}
$$

where $r_{n}^{+}$and $\overrightarrow{r_{n}}$ are the rate of entering and leaving the electron reservoir (gain medium) and $r_{s}^{+}$and $r_{s}^{-}$are the corresponding rates for the photon reservoir (lasing mode). The rates of exchange between electron and photon reservoir are denoted by $r_{n s}$ and $r_{s n}$. Equation (2a) and (2b) has been used to simplify the expressions in (3). The pump term $i_{0} / q$ does not enter directly into ( $3 \mathrm{a})$ in agreement with the collective noise treatment of a semiconductor diode [12]. The reason for the term $E_{V C} s_{0}$ is that the sum of emission and absorption rates contributes to noise, while only the difference enters into the gain expression.

\section{Electrical Equivalent Circuit}

A small-signal analysis of the rate equation (1) gives the following relations for small photon $\left(s_{1}\right)$, electron $\left(n_{1}\right)$, and pump $\left(i_{1}\right)$ variations:

$$
\frac{d n_{1}}{d t}=\frac{i_{1}}{q}-\left[\frac{1}{\tau_{s}}+A s_{0}\right] n_{1}-g_{0} s_{1}+f_{N}
$$

$$
\frac{d s_{1}}{d t}=\left[\frac{\beta}{\tau_{s}}+A s_{0}\right] n_{1}-\beta \frac{n_{0}}{s_{0} \tau_{p h}} s_{1}+f_{S} .
$$

$A$ is defined as differential gain slope $A=d g / d n$. The smallsignal junction voltage $v_{1}$ is introduced by the following boundary condition:

$$
\begin{aligned}
& v_{1}=m V_{T} \frac{n_{1}}{n_{0}} \quad V_{T}=\frac{k T}{q} \\
& m=2+\frac{n_{0}}{\operatorname{Vol} 2 \sqrt{2}}\left[\frac{1}{N_{V}}+\frac{1}{N_{C}}\right] .
\end{aligned}
$$

$m$ in (5b) is given by an approximation of the Fermi-Dirac distribution [13], where $N_{V}$ and $N_{C}$ are the effective valenceand conduction-band densities. $V_{T}$ is the thermal voltage, $k$ is Boltzman's constant, $q$ is the electron charge, and $T$ is the absolute temperature. Anticipating the resulting equivalent electrical circuit of the diode a new variable $i_{L}$ is introduced.

$$
i_{L}=q g_{0} s_{1} \text {. }
$$

Now consider the separate problem of the equivalent circuit shown in Fig. 2. The equations describing $v_{1}$ and $i_{L}$ of the equivalent circuit can be written by inspection.

$$
\begin{aligned}
& \frac{d v_{1}}{d t}=-\frac{v_{1}}{R C}+\frac{i_{1}}{C}-\frac{i_{L}}{C}+\frac{i_{n}}{C} \\
& \frac{d i_{L}}{d t}=\frac{v_{1}}{L}-\frac{R_{s e}}{L} i_{L}-\frac{v_{n}}{L} .
\end{aligned}
$$

Equations (4a), (4b), (7a), and (7b) are identical in form and by comparing constants and using the definitions $(5 a),(5 b)$, and (6), one obtains the values of the equivalent electrical circuit as function of $n_{0}, g_{0}$, and $s_{0}$ given by (2a) and (2b).

$$
\begin{aligned}
C & =\frac{q}{m V_{T}} n_{0} \\
R & =\frac{m V_{T}}{q n_{0}\left[A s_{0}+\left(1 / \tau_{s}\right)\right]} \\
L & =\frac{m V_{T}}{q n_{0} g_{0}\left[A s_{0}+\left(\beta / \tau_{s}\right)\right]} \\
R_{s e} & =\frac{m V_{T} \beta}{q \tau_{s} s_{0} g_{0}\left[A s_{0}+\left(\beta / \tau_{s}\right)\right]} .
\end{aligned}
$$

$C$ is the usual diffusion capacitance resulting from the storage effect of the injected carriers. $R$ is a modified differential diode resistance which approaches the conventional $m V_{T} / i_{0}$ expression in the absence of photons.

In $\mathrm{Fig} .3$ we plot the elements of the equivalent circuit using (8)-(11) with the following laser parameters. Volume of the active region $\mathrm{Vol}=300 \times 5 \times 0.2 \mu \mathrm{m}^{3}$, spontaneous lifetime $\tau_{s}=3 \mathrm{~ns}$, photon lifetime $\tau_{p h}=2 \mathrm{ps}$, gain $g_{0}=A\left(n_{0}-n_{n o m}\right)$, $A=5 \times 10^{-7} \mathrm{~cm}^{3} \cdot \mathrm{s}^{-1} / \mathrm{Vol}=1.7 \times 10^{3} \mathrm{~s}^{-1}, n_{\text {nom }}=5 \times 10^{17}$ $\mathrm{cm}^{-3} \times \mathrm{Vol}=1.5 \times 10^{8}$, and threshold current $i_{\mathrm{th}}=q / \tau_{s}$ $\left[n_{\text {nom }}+\left(1 / A \tau_{p h}\right)\right]=24 \mathrm{~mA}$. The small-signal current in the inductive branch, earlier arbitrarily defined as $i_{L}$ in (6), is directly proportional to the optical signal. 


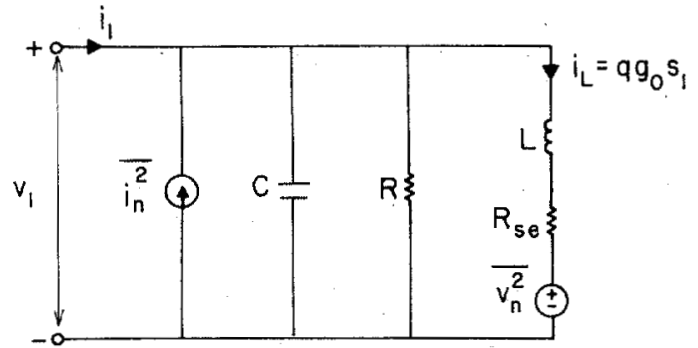

Fig. 2. Small-signal model of a semiconductor laser diode. The input signal is the modulation current $i_{1}$ or the modulation voltage $v_{1}$. The optical output signal is directly proportional to $i_{L}$. The values of the components $R, C, L$, and $R_{s e}$ are given in Fig. 3. The intrinsic shot noise is modeled by the voltage and current noise sources.
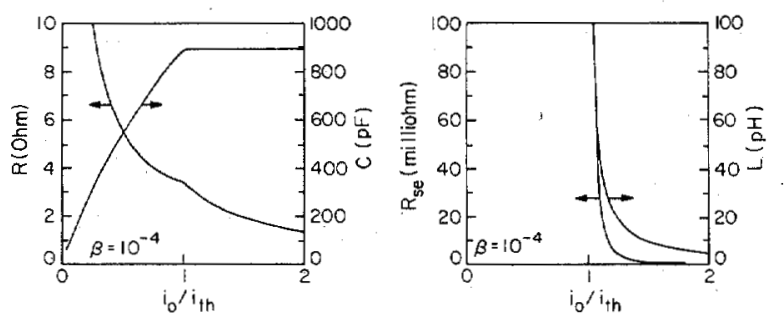

Fig. 3. Values of the elements of the equivalent circuit as a function of the bias point. The parameters of the modeled laser are given in the text.

The spectral intensities $S_{i i}$ and $S_{v v}$ of the current and voltage noise source and the cross spectral intensity $S_{i v}$ do not depend on frequency and are given by comparing the Fourier transforms of (4a), (4b), (7a), and (7b).

$$
\begin{aligned}
S_{i i} & =\frac{\overline{i_{n}^{2}}}{\Delta f}=2 C^{2}\left[\frac{m V_{T}}{n_{0}}\right]^{2}\left\langle F_{N}^{2}(f)\right\rangle \\
& =2 q i_{0}+4 q^{2} E_{V C} s_{0} \\
S_{u v} & =\frac{\overline{v_{n}^{2}}}{\Delta f}=2 L^{2}\left[q g_{0}\right]^{2}\left\langle F_{S}^{2}(f)\right\rangle \\
& =4 \frac{\left[m V_{T}\right]^{2}}{\left[n_{0}\left[A s_{0}+\left(\beta / \tau_{s}\right)\right]\right]^{2}}\left[\frac{1}{\tau_{p h}}+E_{V C}\right] s_{0} \\
S_{\dot{w}} & =\frac{\overline{i_{n} v_{n}}}{\Delta f}=-2 L C \frac{m V_{T}}{n_{0}} q g_{0}\left\langle F_{N}(f) F_{S}(f)\right\rangle \\
& =2 \frac{m V_{T} q}{n_{0}\left[A s_{0}+\left(\beta / \tau_{s}\right)\right]}\left[\frac{1}{\tau_{p h}}+2 E_{V C}\right] s_{0} .
\end{aligned}
$$

An additional factor of 2 has been included because we changed from the double-sided to the more common singlesided noise spectrum. The current noise source $i_{n}$ in parallel with the junction represents mainly the fluctuation in electron population. The first term on the right side of (12a) is the usual shot noise term $2 q i_{0}$ of a nonlasing diode. The second term is resulting from the fact that the noise is determined by the sum of emission and absorption, whereas the net gain corresponds to the difference of the rates.

The fluctuation of the photon population is due mainly to the shot noise of the gain mechanism, that is, due to the voltage noise source $v_{n}$. The sources $i_{n}$ and $v_{n}$ are partially
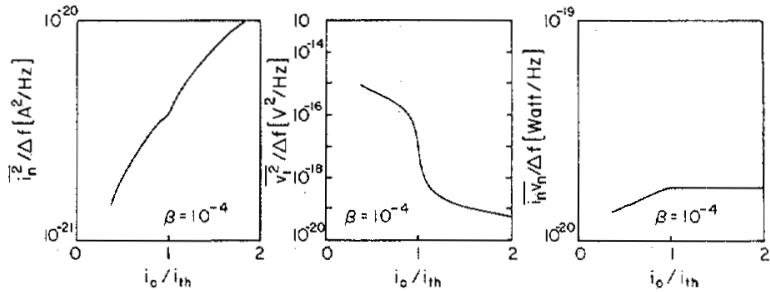

Fig. 4. Spectral intensities of the noise sources of the equivalent circuit as a function of the normalized bias current $i_{0} / i_{\text {th }}$.

correlated due to the coupled rates and their cross spectral intensity is given by (12c). The spectra of the noise sources $\overline{i_{n}^{2}} / \Delta f, \overline{v_{n}^{2}} / \Delta f$, and $\overline{i_{n} v_{n}} / \Delta f$, given by (12a)-(12c) are plotted in Fig. 4 as a function of the pump current, where a simple model has been used to calculate $E_{V C}$.

\section{An Application of the Formalism}

From the intrinsic equivalent circuit and the knowledge of the extrinsic parameters (series resistance, parasitic capacitance, and inductance, the impedance of the modulating source) the noise and modulation characteristics of the laser diode can be calculated. We limit ourselves in the following example to the intrinsic case and current modulation. The following properties of the laser diode are calculated: $Z(\omega)$, the diode impedance, $H(\omega)$, the photon modulation transfer function, $\overline{v_{1}^{2}} / \Delta f$, the junction voltage noise spectrum, and $\mathrm{RIN} / \Delta f$, the relative intensity noise spectrum.

$$
\begin{aligned}
Z(\omega) & =\frac{V_{1}(\omega)}{I_{1}(\omega)}=\frac{1}{L C} \frac{\left[R_{s e}+j \omega L\right]}{D} \\
H(\omega) & =\frac{S_{1}(\omega)}{I_{1}(\omega)}=\frac{1}{L C q g_{0} D} \\
\frac{\overline{v_{1}^{2}}(\omega)}{\Delta f} & =\frac{\overline{i_{n}^{2}}\left[R_{s e}^{2}+[\omega L]^{2}\right]+\overline{v_{n}^{2}}+2 \overline{v_{n} i_{n}} R_{s e}}{[L C]^{2} D D^{*}} \frac{1}{\Delta f} \\
\frac{\operatorname{RNN}(\omega)}{\Delta f} & =\frac{S_{s}(\omega)}{s_{0}^{2}} \\
& =\frac{\overline{i_{n}^{2}}+\overline{v_{n}^{2}}\left[\left(1 / R^{2}\right)+[\omega C]^{2}\right]-2 \overline{v_{n} i_{n}}(1 / R)}{[L C]^{2} D D^{*}\left[q g_{0}\right]^{2}} \frac{1}{\Delta f} \\
D & =-\omega^{2}+j \omega\left[\frac{R_{s e}}{L}+\frac{1}{R C}\right]+\frac{1}{L C}\left[1+\frac{R_{s e}}{R}\right] \quad(13 \mathrm{e}) \\
S_{s}(\omega) & =2 \int_{-\infty}^{\infty}\left[\int_{-\infty}^{\infty} s(t) s(t+\tau) d t\right] e^{-j \omega \tau} d \tau
\end{aligned}
$$

where $S_{s}(\omega)$ is the photon noise spectrum. Figs. 5 and 6 show the calculated dependence of $v_{1}^{2} / \Delta f$ and RIN/ $/ \Delta f$ on current and frequency, and display the characteristic noise peak at onset of lasing and the high-frequency resonance. The frequency dependence of $Z(\omega)$ is shown in Fig. 7 for different pump levels. 

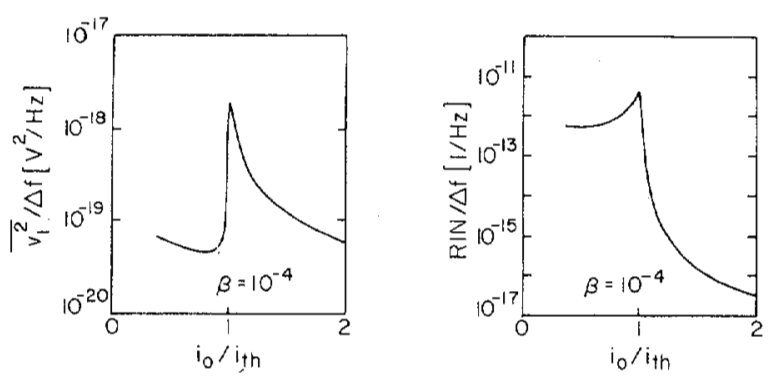

Fig. 5. The junction voltage noise spectrum $\overline{v_{1}^{2}} / \Delta f$ of a laser diode driven by a current source at low frequencies as a function of the normalized bias current $i_{0} / i_{\text {th }}$. RIN/ $\Delta f$ is the relative intensity noise spectrum. Both noise spectra show a peak at the onset of stimulated emission.
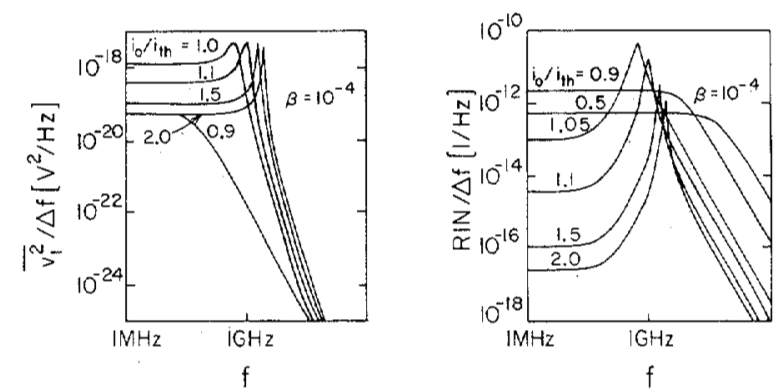

Fig. 6. Frequency dependence of the junction voltage noise spectrum $\left(\overline{v_{1}^{2}} / \Delta f\right)$ and the relative intensity noise spectrum (RIN/ $\left./ \Delta f\right)$ as a function of the normalized bias current $i_{0} / i_{\text {th }}$. Both spectra show a resonance at the relaxation frequency of the laser.

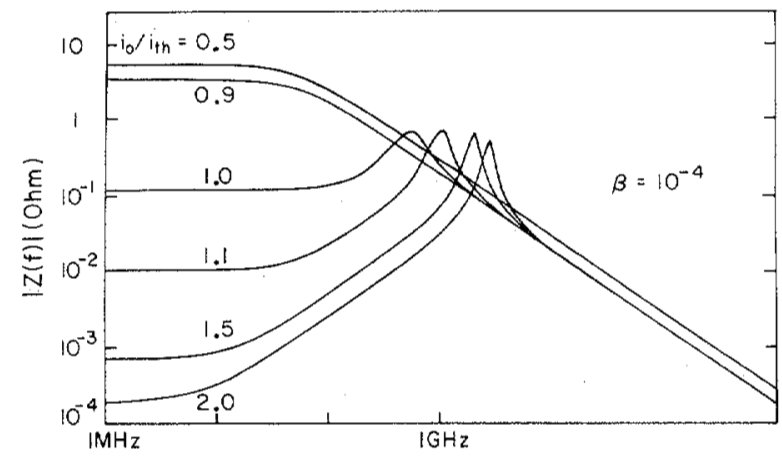

Fig. 7. Frequency dependence of the impedance of a diode laser as function of the normalized bias current $i_{0} / i_{\mathrm{th}}$.

\section{Noise of the Multilongitudinal Mode Laser}

The rate equations for a laser emitting radiation into $n$ longitudinal modes are

$$
\begin{aligned}
& \frac{d n}{d t}=\frac{i}{q}-\sum_{k=1}^{k=n} g_{k} s_{k}-\frac{n}{\tau_{s}}+f_{N} \\
& \frac{d s_{k}}{d t}=g_{k} s_{k}-\frac{s_{k}}{\tau_{p h k}}+\beta_{k} \frac{n}{\tau_{s}}+f_{S k} \quad k=1 \cdots n .
\end{aligned}
$$

An analysis analogous to that above yields the equivalent circuit shown in Fig. 8. Each optical mode contributes a parallel branch to the circuit with a current $i_{L k}$ corresponding to the photons of the signal in the mode $k$. The capacitance modeling the injected carriers remains unchanged. Taking the

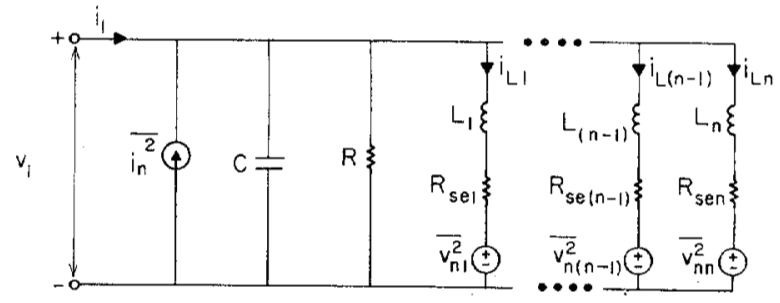

Fig. 8. Noise equivalent circuit of a multilongitudinal semiconductor laser diode. Each optical mode contributes a parallel branch to the circuit with a current $i_{L k}$ proportional to the photons of the signal in the mode $k$.

gain of the $k$ th mode as $g_{k}=g_{0 k}+A_{k} n_{1 k}$ the values of the components are

$$
\begin{aligned}
R & =\frac{m V_{T}}{q n_{0}} \frac{1}{\sum_{k=1}^{k=n} A_{k} s_{0 k}+\left(1 / \tau_{s}\right)} \\
L_{k} & =\frac{m V_{T}}{q n_{0}} \frac{1}{g_{0 k}\left[A_{k} s_{0 k}+\left(\beta_{k} / \tau_{s}\right)\right]} \\
R_{\text {sek }} & =\frac{\beta n_{0}}{\tau_{s} s_{0}} \frac{m V_{T}}{q n_{0}} \frac{1}{g_{0 k}\left[A_{k} s_{0 k}+\left(\beta_{k} / \tau_{s}\right)\right]} .
\end{aligned}
$$

The noise power spectra are:

$$
\begin{gathered}
\frac{\overline{i_{n}^{2}}}{\Delta f}=2 q i_{0}+4 q^{2} \sum_{k=1}^{k=n} E_{V C k} s_{0 k} \\
\frac{\overline{v_{n k}^{2}}}{\Delta f}=4 \frac{\left[m V_{T}\right]^{2}}{\left[n_{0}\left[A_{k} s_{0 k}+\left(\beta_{k} / \tau_{s}\right)\right]\right]^{2}}\left[\frac{1}{\tau_{p h k}}+E_{V C k}\right] s_{0 k} \\
\frac{\overline{v_{n k} i_{n}}}{\Delta f}=2 \frac{m V_{T} q}{n_{0}\left[A_{k} s_{0 k}+\left(\beta_{k} / \tau_{s}\right)\right]}\left[\frac{1}{\tau_{p h k}}+2 E_{V C k}\right] s_{0 k} \\
\quad \overline{v_{n m} v_{n l}}=0 \quad m \neq l .
\end{gathered}
$$

This noise analysis of a multimode laser neglecting the phase relations between the modes is justified if the beat frequencies of the longitudinal modes lie outside the spectrum of interest. The model above predicts that the relative intensity fluctuations of one isolated mode of a multimode laser are several orders of magnitude larger than the relative intensity fluctuations of all the modes together [8]. The intensity fluctuations of different modes are negatively correlated because the different modes are competing for gain from the same electron reservoir. This so-called competition noise may significantly deteriorate the signal-to-noise ratio in communication systems which have mode selective elements, such as material dispersion or interference effects [10].

\section{CONCLUSIONS}

The small-signal model of a semiconductor laser has been extended to include the effects of the intrinsic noise by adding a current and a voltage noise sources. The current noise source represents the shot noise of the recombination of the carriers and the voltage noise source the random process of stimulated 
emission. The intensity fluctuation of the light is dominated by the stimulated emission process. The usefulness of the noise equivalent circuit in modeling an electronic circuit with an injection laser as one of its elements is demonstrated by calculating the modulation and noise characteristic of a current driven laser diode. In addition, the model has been generalized to the multimode case.

\section{REFERENCES}

[1] M. Morishita, T. Ohmi, and J. Nishizawa, "Impedance characterics of double-heterostructure laser diodes," Solid-State Electron., vol. 22, pp. 951-962, Nov. 1979.

[2] J. Katz, S. Margalit, C. Harder, D. Wilt, and A. Yariv, "The intrinsic equivalent circuit of a laser diode," IEEE $J$. Quantum Electron., vol. QE-17, pp. 4-7, Jan. 1981.

[3] D. E. McCumber, "Intensity fluctuations in the output of $\mathrm{cw}$ laser oscillators. I," Phys. Rev., vol. 141, pp. 306-322, Jan. 1966.

[4] M. Lax, "Quantum noise VII: The rate equations and amplitude noise in lasers," IEEE J. Quantum Electron., vol. QE-3, pp. 3746, Feb. 1967.

[5] H. Haug, "Quantum-mechanical rate equations for semiconductor lasers," Phys. Rev., vol. 184, pp. 338-348, Aug. 1969.

[6] D. J. Morgan and M. J. Adams, "Quantum noise in semiconductor lasers," Phys. Status Solidi: (a), vol. 11, pp. 243-253, 1972.

[7] T. L. Paoli and J. E. Ripper, "Observation of intrinsic quantum fluctuations in semiconductor lasers," Phys. Rev. A., vol. 2, pp. 2551-2555, 1970.

[8] H. Jackel, "Light intensity fluctuations and dynamic behaviour of GaAlAs heterostructure diode lasers in the frequency range of $10 \mathrm{MHz}$ to $8 \mathrm{GHz}$," Ph.D. dissertation, ETH Nr. 6447, Zurich, Switzerland, 1980.

[9] H. Melchior, "Noise in semiconductor lasers," in Top. Meet. Integrated and Guided-Wave Opt., Jan. 1980, paper MA2.

[10] G. Arnold and K. Petermann, "Intrinsic noise of semiconductor lasers in optical communication systems," Optical and Quantum Electron., vol. 12, pp. 207-219, 1980.

[11] S. O. Rice, "Mathematical analysis of random noise," Bell Syst. Tech. J., vol. 23, pp. 282-332, July 1944; and Bell Syst. Tech. J., vol. 24, pp. 46-156, Jan. 1945.

[12] A. Van der Ziel, Fluctuation Phenomena in Semiconductors. London: Butterworth, 1959.

[13] W. B. Joyce and R. W. Dixon, "Analy tic approximations for the Fermi energy of an ideal Fermi gas," Appl. Phys. Lett., vol. 31, pp. 354-356, Sept. 1977.

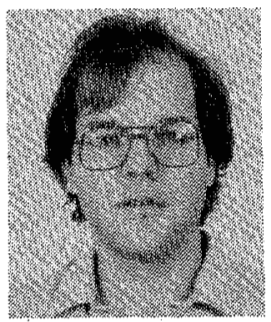

Christoph Harder was born in Rheinau, Switzerland, on September 3, 1953. He received the Dipl.-Ing. degree in electrical engineering from the Eidgenossische Technische Hochschule (ETH), Zurich, Switzerland, in 1978. In 1980 he came to the California Institute of Technology, Pasadena, on a Fulbright Scholarship, where he received the M.S. degree in electrical engineering. He is currently working towards the Ph.D. degree in the field of semiconductor lasers at the California Institute of Technology.

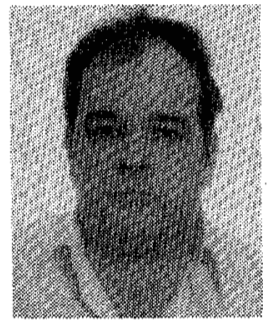

Joseph Katz was born in Tel-Aviv, Israel, on June 6,1952 . He received the B.S. degree from the Technion-Israel Institute of Technology, Haifa, Israel, in 1973, the M.S. degree from TelAviv University, Tel-Aviv, Israel, in 1976, and the Ph.D. degree from the California Institute of Technology, Pasadena, in 1981, all in electrical engineering.

In June 1981 he joined the Jet Propulsion Laboratory at the California Institute of Technology, Pasadena, where he is currently working in the field of optical communications.

S. Margalit, photograph and biography not available at the time of publication.

J. Shacham, photograph and biography not available at the time of publication.

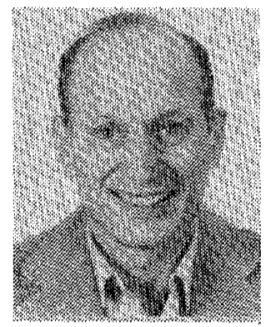

Amnon Yariv (S'56-M'59-F'70) was born in Tel-Aviv, Israel, on April 13, 1930. He received the B.S., the M.S., and the Ph.D. degrees all in electrical engineering, from the University of California, Berkeley, in 1954, 1956, and 1958, respectively.

In 1959 he went to Bell Laboratories, Murray Hill, NJ, where he joined the early stages of the laser effort. In 1964 he joined the California Institute of Technology, Pasadena, as an Associate Professor of Electrical Engineering, and in 1966 he became a Professor. In 1980 he became the Thomas G. Myers Professor of Electrical Engineering and Applied Physics. On the technical side, he took part (with various co-workers), in the discovery of a number of early solid-state laser systems, the formulation of the theory of parametric quantum noise and the prediction of parametric fluorescence, the invention of the technique of mode-locked ultrashortpulse lasers and FM lasers, the introduction of $\mathrm{GaAs}$ and CdTe as infrared electrooptic and window materials, proposing and demonstrating semiconductor based integrated optics technology, and pioneering the field of phase conjugate optics. His present research efforts are in the areas of nonlinear optics, recombination mechanisms in semiconductors, semiconductor lasers, and integrated optics, especially the problem of monolithic integration of transistors, injection lasers, and detectors for high-frequency applications. He authored or coauthored some 190 papers in professional journals, as well as two books: Quantum Electronics (New York: Wiley 1967, 1975) and Introduction to Optical Electronics (New York: Holt, Rinehart, and Winston, 1971, 1976).

$\mathrm{He}$ is also the Associate Editor of Optics Communications and the Journal of Applied Physics, and was previously Associate Editor of the IEEE Journal of QUANTUM Electronics. Dr. Yariv is a member of the American Physical Society, Phi Beta Kappa, and the National Academy of Engineering, and a Fellow of the Optical Society of America. He is a consultant to the Hughes Aircraft Research Laboratories and is the recipient of the 1980 Quantum Electronics Award of the IEEE. 